\title{
Nucleation and Growth of Zinc Sulfide Nanoparticles in Ultrathin Polymer Films by Layer-by-Layer Polyionic Assemblies
}

\author{
Sevil Cetinkaya ${ }^{1,2}$, Qian Zhou ${ }^{1,3}$, Shuangjian Zhang ${ }^{1}$, Ashkon Mohebi ${ }^{1}$, Dat V. Quach ${ }^{1}$, Pieter Stroeve ${ }^{1}$ \\ ${ }^{1}$ Department of Chemical Engineering and Materials Science, University of California Davis, Davis, USA; ${ }^{2}$ Cumhuriyet University, \\ Faculty of Engineering, Department of Chemical Engineering, Sivas, Turkey; ${ }^{3}$ State Key Laboratory of Heavy Oil Processing, China \\ University of Petroleum, Beijing, China. \\ Email: pstroeve@ucdavis.edu
}

Received February $16^{\text {th }}, 2011$; revised February $28^{\text {th }}, 2011$; accepted March $9^{\text {th }}, 2011$.

\begin{abstract}
In this work, zinc sulfide $(\mathrm{ZnS})$ nanoparticles were formed by nucleation and growth in ultrathin films of polydiallyldimethylammonium chloride (PDDA)-polystyrenesulfonate sodium salt (PSS) film produced by the Layer-by-Layer (LbL) deposition technique. Multilayer thin film assemblies, fabricated by sequential adsorption of polyelectrolytes on a quartz substrate, were used as a supramolecular reaction template to study the in-situ nucleation and growth of ZnS nanoparticles. ZnS nanoparticles were nucleated within the polymeric supramolecular structure through cyclic exposure to the solutions of $\mathrm{Zn}\left(\mathrm{NO}_{3}\right)_{2}$ and thiourea. The growth and nucleation of nanoparticles were accomplished by a cyclic repetition of reductive hydrolysis reactions. The growth of a thin film on a flat substrate via LbL was monitored by ultraviolet-visible (UV-Vis) spectroscopy. Analysis of the UV-visible absorption spectra of the films revealed that the nanoparticles grew with increasing number of cycles. The presence of ZnS nanoparticles were verified by transmission electron microscopy (TEM). Selected area electron diffraction (SAED) showed that the ZnS has a cubic spheralite structure.
\end{abstract}

Keywords: Layer-by-Layer, Nanoparticles, Zinc Sulfide, Reducing Reaction, In-Situ Nucleation

\section{Introduction}

Zinc sulfide $(\mathrm{ZnS})$ nanoparticles have recently received significant attention because of their unique optical, electrical and magnetic properties [1]. $\mathrm{ZnS}$ is one of the most important wide band gap II-VI semiconductor materials, which has many valuable properties such as photoluminescence, electroluminescence and photocatalytic properties. Because of these properties, $\mathrm{ZnS}$ nanoparticles can be used in various applications including optoelectronic devices, electroluminescent devices, and photovoltaic cells $[2,3]$. Several techniques on growing $\mathrm{ZnS}$ thin films such as chemical vapor deposition (CVD) [4], chemical bath deposition (CBD) [5], and close spaced evaporation (CSE) technique [6] have been reported.

Over the last few decades, an increased interest in the field of polyelectrolyte deposition and self-assembling methods of macromolecules and nanoparticles [7-9] has been reported. The Layer-by-Layer (LbL) deposition tech- nique of building supramolecular multilayers on solid substrates by adsorbing polyelectrolytes has many advantages due to its simplicity, low cost and ease at controlling template's thickness. There have been investigations on the growth of gold/zinc sulfide [10], nickel hydroxide [11], cobalt hydroxide [12], lepidocrocite [13], lead sulfide [14] and iron oxy-hydroxide nanoparticles [15] using the LbL technique. Stroeve and coworkers [13, 15] have demonstrated that divalent and trivalent ions can bind to the negatively charged sulfonate groups in LbL polyelectrolyte nanofilms.

This method manufactures precursor polyelectrolyte multilayer films through LbL technique to form nanoreactors, and then nucleation of nanoparticles is initiated by adsorption and hydrolysis of metal ions. Once nuclei form, the growth of nanoparticles can be controlled by the number of the adsorption and hydrolysis cycles [16]. Some researchers have employed polyelectrolyte multilayer films as nanoreactors for preparing nanoparticles 
with controlled size via manipulating multilayer processing conditions [17]. Logar and coworkers [18] have investigated the growth characteristics of $\mathrm{ZnS}$ nanocrystallites within the weak polyelectrolyte multilayer (PEM) assemblies and correlated with the optical properties of the $\mathrm{ZnS} / \mathrm{PEM}$ composite films, for physical properties of semiconducting nanoparticles are affected by their size, morphology and crystal structure. There has been no report so far on the formation of $\mathrm{ZnS}$ nanoparticles embedded in multilayer films by LbL technique. The ability to embed $\mathrm{ZnS}$ nanoparticles in a thin film is desirable because the LbL technique can be used as a coating procedure on substrates with either flat, curved or complex surfaces.

Because of the importance of $\mathrm{ZnS}$ as a wide band gap IIVI semiconductor materials, in this work, zinc sulfide (ZnS) nanoparticles were formed by nucleation and growth in polydiallyldimethylammonium chloride (PDDA)-polystyrenesulfonate sodium salt (PSS) film produced by LbL deposition technique. Multilayer thin film assemblies, fabricated by the sequential adsorption of polyelectrolytes on a quartz substrate, served as a supramolecular reaction template to study the in-situ nucleation and growth of $\mathrm{ZnS}$ nanoparticles. $\mathrm{ZnS}$ nanoparticles were nucleated within the polymeric supramolecular structure through cyclic exposure to first $\mathrm{Zn}\left(\mathrm{NO}_{3}\right)_{2}$ and then to thiourea solutions. The films and nanoparticles there-in were analyzed with nanoparticles.

\section{Experimental}

PDDA and PSS were purchased from Polyscience Inc. and used as received. Sodium hydroxide, hydrochloric acid and $29.5 \%$ ammonium solution $\left(\mathrm{NH}_{4} \mathrm{OH}\right)\left(\mathrm{NH}_{3} \cdot \mathrm{H}_{2} \mathrm{O}\right)$ were products of Fisher Scientific. 99.99\% zinc nitrate hydrate $\left(\mathrm{Zn}\left(\mathrm{NO}_{3}\right)_{2} \cdot 6 \mathrm{H}_{2} \mathrm{O}\right), 99 \%$ thiourea $\left(\mathrm{CS}\left(\mathrm{NH}_{2}\right)_{2}\right)$, 98\% hydrazine anhydrous $\left(\mathrm{N}_{2} \mathrm{H}_{4}\right)$ were obtained from Aldrich, Acros, and Sigma, respectively. All solutions were prepared in purified water from a Nanopure water purification system (Bransted).

The quartz slides were used as substrates for UV-Vis measurements and were cleaned as follows: 1) ultrasonication in deionized water $\left(\right.$ at $50^{\circ} \mathrm{C}$ ) for $\left.15 \mathrm{~min}, 2\right)$ ultrasonication with Helmanex solution $\left(2 \%\right.$, at $\left.50^{\circ} \mathrm{C}\right)$ for 15 min, 3) rinsed with purified water thoroughly after each ultrasonication, 4) ultrasonication of the slide in pure ethanol $\left(\right.$ at $50^{\circ} \mathrm{C}$ ) for $15 \mathrm{~min}$.

The PDDA and PSS polymer solutions were both prepared in $20 \mathrm{mM}$ concentrations. All concentrations were calculated based on the monomer molecular weights. The PDDA solution was dissolved in deionized water, while the PSS solution was dissolved in a $0.1 \mathrm{M} \mathrm{NaOH}$ solution and then adjusted to a $\mathrm{pH}$ range of $4.45-4.55$ by drop wise addition of $0.1 \mathrm{M}$ HCI. The polymer films were obtained by dipping the slides first in the polycation (PDDA) solution for $20 \mathrm{~min}$, then rinsing with deionized water, and finally treating with $0.1 \mathrm{M} \mathrm{HCI}$ to charge the surface layer. The slides were then dried completely by a stream of nitrogen. After this process, the slides were dipped in the polyanion (PSS) solution for $20 \mathrm{~min}$. This method allows multilayer polymer films to grow on the quartz surface in a stable and reproducible manner [7, 19]. In the experiments PDDA was chosen as the outer layer since particle growth will also be on the outside of the film rather than inside of the film. With PDDA as the final layer, the $\mathrm{ZnS}$ nucleation and growth is exclusively inside the LbL film, since the reactive sites are the sulfonate groups of the PSS layers [15].

The in-situ nucleation of $\mathrm{ZnS}$ particles within the PDDA-PSS film was obtained by first absorbing $\mathrm{Zn}^{2+}$ ions into the polymer film by dipping it for $5 \mathrm{~min}$ in the first solution containing $\mathrm{Zn}\left(\mathrm{NO}_{3}\right)_{2} \cdot 6 \mathrm{H}_{2} \mathrm{O}\left(0.077 \mathrm{~mol} \cdot \mathrm{L}^{-1}\right)$, ammonia $\left(1.39 \mathrm{~mol} \cdot \mathrm{L}^{-1}\right)$ and hydrazine anhydrous $(2.29$ $\left.\mathrm{mol} \cdot \mathrm{L}^{-1}\right)$. Next, the film was rinsed with water thoroughly to remove extra $\mathrm{Zn}^{2+}$ from the film surface and then dried with nitrogen gas before exposed to the second solution. The second solution was composed of aqueous solution of ammonia $\left(1.39 \mathrm{~mol} \cdot \mathrm{L}^{-1}\right)$ and thiourea $(0.071$ $\left.\mathrm{mol} \cdot \mathrm{L}^{-1}\right)$. In the next step, the film was dipped in the second solution for $5 \mathrm{~min}$ to initiate the formation of $\mathrm{ZnS}$ particles within the film. Finally, the films were submerged in water for $1 \mathrm{~min}$ and then dried with nitrogen gas. At this point, the film had gone through one reaction cycle. The UV-visible spectra were obtained after each reaction cycle before $\mathrm{HCl}$ treatment.

Thiourea provides the necessary $\mathrm{S}^{-2}$ ions by hydrolysis. The various reactions involved in this growth process are given in the following [20,21]:

$$
\begin{aligned}
\mathrm{NH}_{3}+\mathrm{H}_{2} \mathrm{O} \Leftrightarrow \mathrm{NH}_{4}^{+}+\mathrm{OH}^{-} \\
\mathrm{N}_{2} \mathrm{H}_{4}+\mathrm{H}_{2} \mathrm{O} \Leftrightarrow \mathrm{N}_{2} \mathrm{H}_{5}^{+}+\mathrm{OH}^{-} \\
\mathrm{Zn}\left(\mathrm{NO}_{3}\right)_{2} \rightarrow \mathrm{Zn}^{2+}+2 \mathrm{NO}_{3}
\end{aligned}
$$

$\mathrm{Zn}^{2+}$ ions form the metal complexes with ammonia and hydrazine hydrate by following reaction:

$$
\begin{gathered}
\mathrm{Zn}^{2+}+4 \mathrm{NH}_{3} \Leftrightarrow \mathrm{Zn}\left(\mathrm{NH}_{3}\right)_{4}^{2+} \\
\mathrm{Zn}^{2+}+3\left(\mathrm{~N}_{2} \mathrm{H}_{4}\right) \Leftrightarrow \mathrm{Zn}\left(\mathrm{N}_{2} \mathrm{H}_{4}\right)_{3}^{2+}
\end{gathered}
$$

$\mathrm{Zn}\left(\mathrm{NO}_{3}\right)_{2}$ is used as $\mathrm{Zn}^{2+}$ source, the cation donor, and thiourea is used as $\mathrm{S}^{2-}$ source, the anion donor through hydrolysis in the alkaline medium:

$$
\mathrm{SC}\left(\mathrm{NH}_{2}\right)_{2}+2 \mathrm{OH}^{-} \Leftrightarrow \mathrm{S}^{2-}+\mathrm{CH}_{2} \mathrm{~N}_{2}+2 \mathrm{H}_{2} \mathrm{O}
$$

Finally, zinc sulfide nanoparticles were formed inside the polymer film driven by the static electronic attraction according to the reaction: 


$$
\mathrm{Zn}^{2+}+\mathrm{S}^{2-} \Leftrightarrow \mathrm{ZnS}
$$

For TEM purposes, PDDA-PSS polymer film and $\mathrm{ZnS}$ nanoparticles were grown directly on Formvar TEM grids (mesh size $=300$ ) instead of quartz substrates following the exact procedures as described above. In our study, 6.5 layer pairs of PDDA-PSS were used for UV-Vis and TEM measurements with preferred PDDA as the outer layer.

UV-visible absorption spectra of the thin films deposited on the quartz slides were recorded on a CARY-13 absorption spectrophotometer. Transmission electron microscopy (TEM) of the polymer films deposited on conventional electron microscope copper grids coated with Formvar was done using a Philips CM-12 operation at $100 \mathrm{kV}$.

\section{Results and Discussion}

The LbL technique was used to introduce the polymer films on the quartz slides as shown in Figure 1. UVvisible spectroscopy was employed to monitor both the $\mathrm{LbL}$ adsorption process and the reaction cycles in the polymer films. The figures for the UV-visible absorption changes are shown in Figure 2(a) for the LbL deposition method. Figure 2(a) shows UV-visible spectra of LbL films of PDDA and PSS that is from 1.5 to 6.5 layer pairs (LP) thick deposited on quartz slides. One layer pair is equivalent to one layer of PDDA and one layer of PSS. When there are 1.5 layer pairs the deposition on the quartz slides is in the order of PDDA-PSS-PDDA. In this case there are three layers, with only one layer of PSS sandwiched between to PDDA layers. Figure 2(a) shows that the LbL polymer films have an absorption peak at a wavelength of $225 \mathrm{~nm}$, which is an indicator of the aromatic rings present in the PSS layers since PDDA does not have UV absorption in the UV due to the absence of aromatic rings in PDDA [11,22]. For Figure 2(a) the
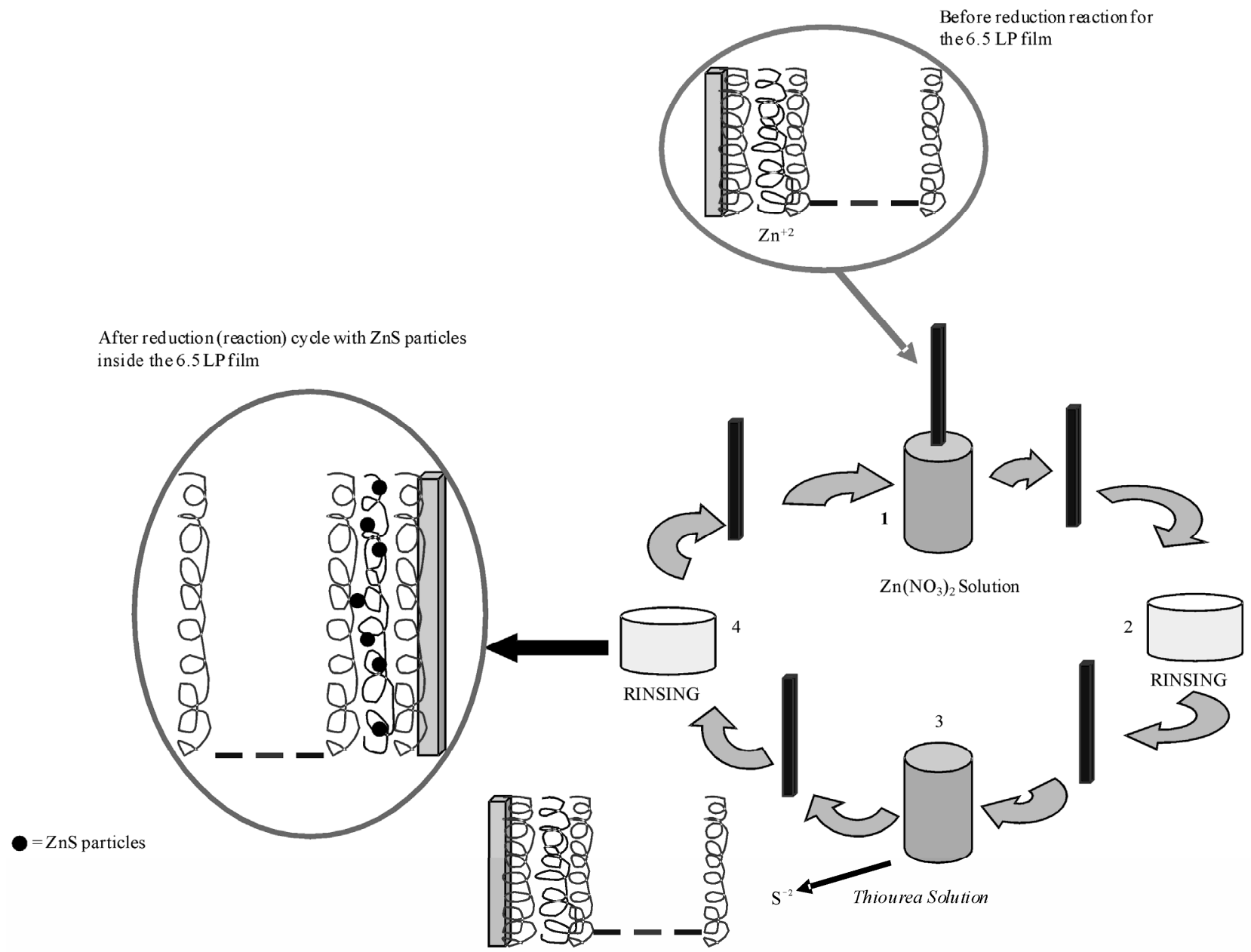

Figure 1. Schematic presentation of PDDA-PSS multilayer film with 6.5 layer pairs for ZnS particle growth by reduction. 


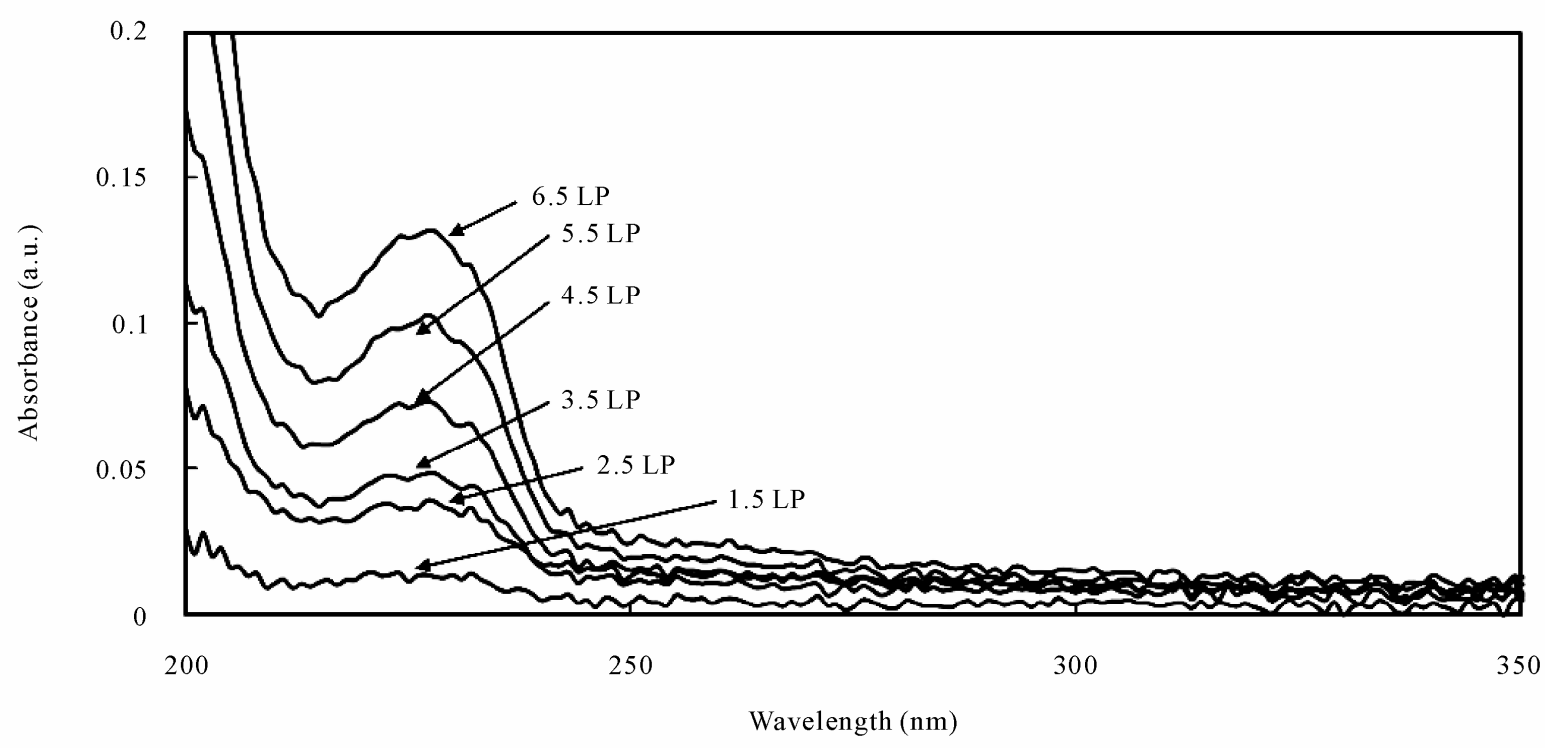

(a)

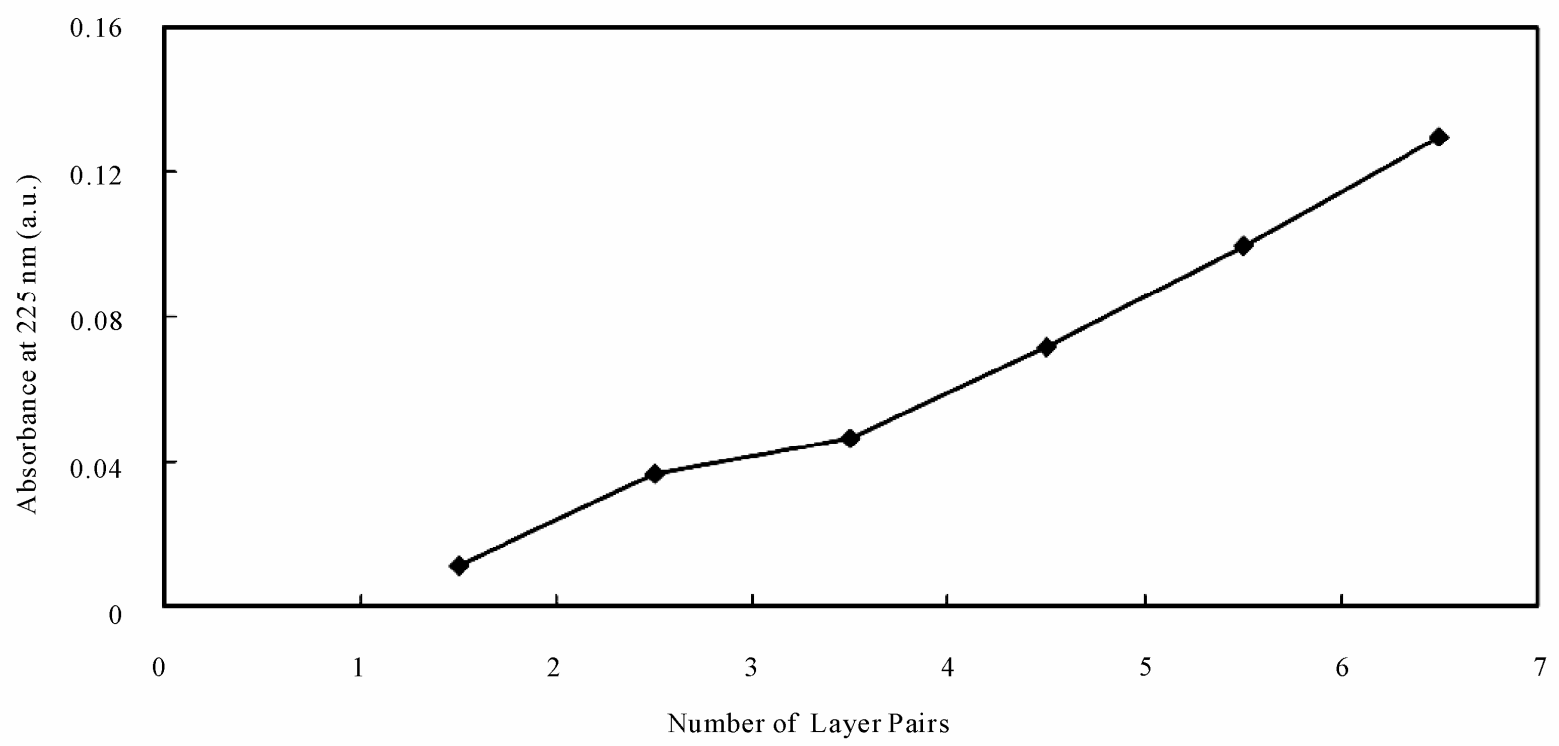

(b)

Figure 2. (a) UV-vis absorption as a function of a number of layer pairs (LP) depositions (without reaction cycles). (b) Absorbance at $225 \mathrm{~nm}$ with respect to the number of layer pairs deposited.

absorption spectra for the six samples are from one (1.5 LP) to six (6.5 LP) layers of PSS. The absorption at 225 $\mathrm{nm}$ increases linearly with the number of layer pairs deposited as seen in Figure 2(b). Thus Figure 2(a) also indicates the sequential increase of the absorption in the UV for each additional PSS layer. Since the increase in the absorption at $225 \mathrm{~nm}$ is nearly linear this indicates that the deposition of PSS on PDDA is uniform.

Nucleation and growth of $\mathrm{ZnS}$ nanoparticles were mo- nitored by UV-visible spectroscopy. Figure 3(a) shows the absorption spectra of an LbL film with 6.5 LP exposed to 5,10,15 and 20 reaction cycles. Each reaction cycles consists of first absorbing $\mathrm{Zn}^{2+}$ ions into the $\mathrm{LbL}$ film. As described in the experimental section, the $\mathrm{Zn}^{2+}$ ions bind to the sulfonate groups in the film, and then the absorbed zinc ions are reduced with thiourea to form $\mathrm{ZnS}$. As can be seen clearly seen in Figure 3(a), an increase in the number of reaction cycles gives an increase in the 


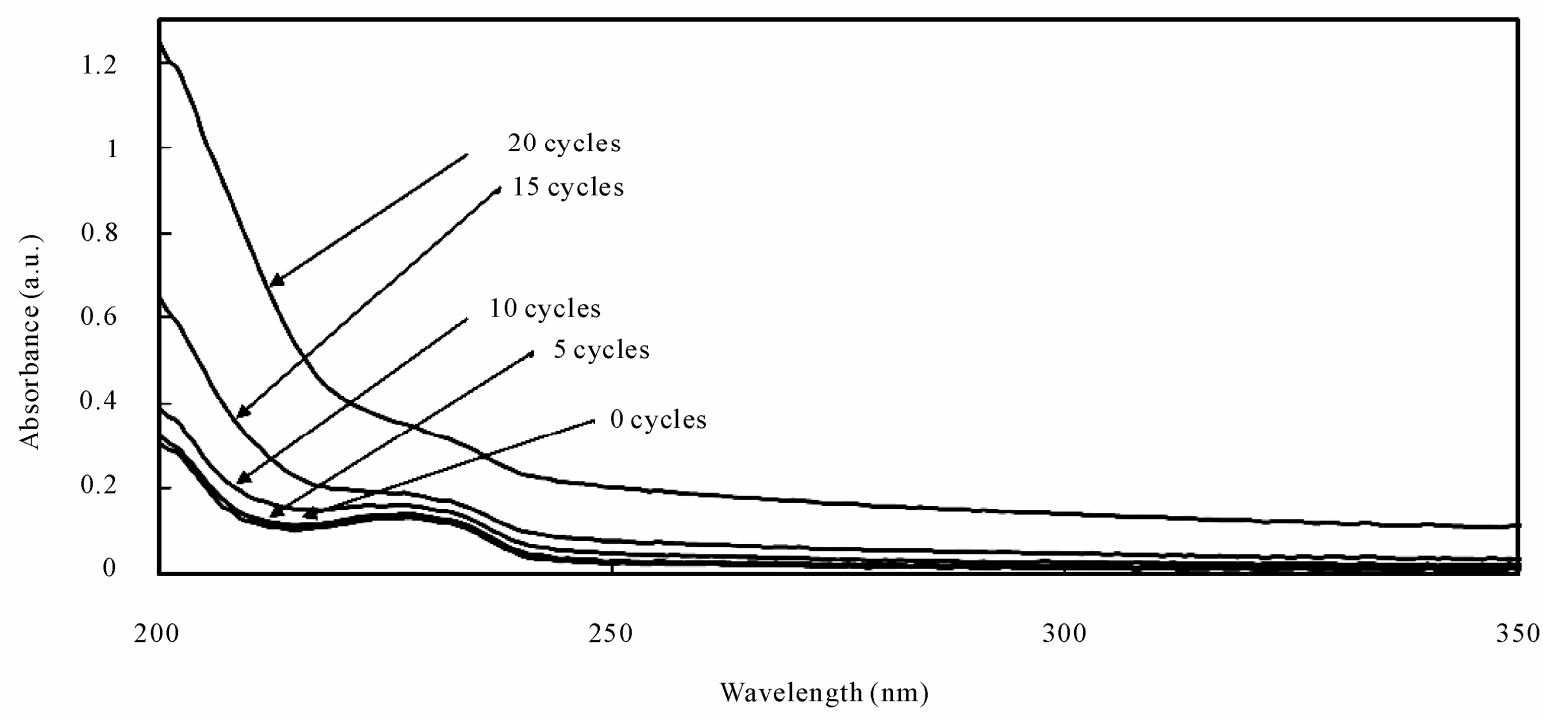

(a)

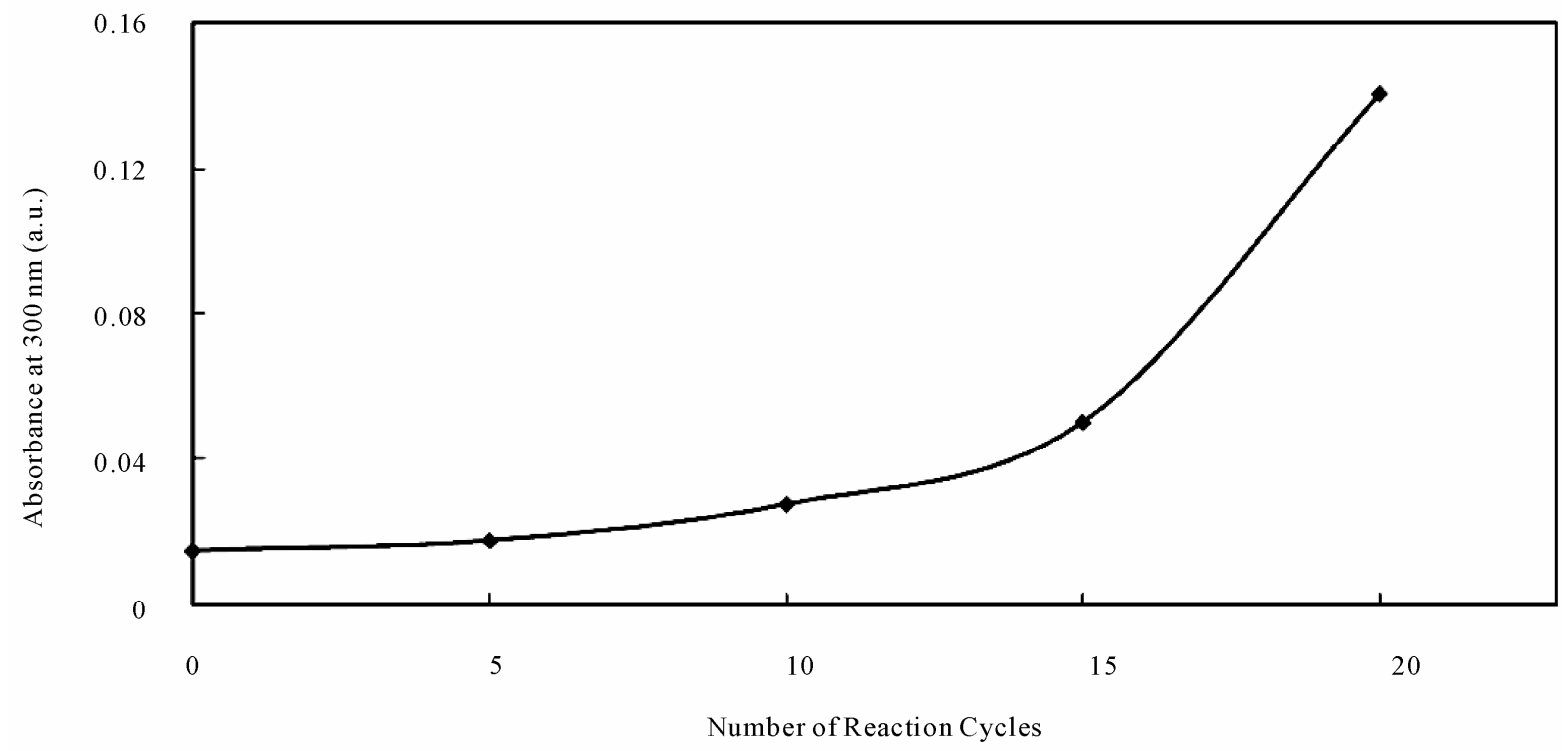

(b)

Figure 3. (a) UV-vis absorption for reaction cycles up to 20 with 6.5 PDDA-PSS layer pairs. (b) Absorbance at $300 \mathrm{~nm}$ with respect to the number of reaction cycles.

absorbance of the film. The spectral profile is now completely different that of the pristine LbL film. The changes in the spectra indicate the formation of zinc sulfide nanoparticles in the film $[12,13]$. The increase of absorbance is with the number of cycles is observed over the whole range of wavelengths. Figure $3 b$ shows that the absorbance for the zinc sulfide nanoparticles in the LbL film with the number of reductive hydrolysis cycles is initially linear for a small number of reduction reaction cycles, but as the number of cycles is increased the response is nonlinear. The data at $300 \mathrm{~nm}$ were chosen to denote the change in absorption for 6.5 layer-pair film in the visible range $[11,12,14]$, although the same results are observed if the data are plotted at other wavelengths such as 350 $\mathrm{nm}$ or $400 \mathrm{~nm}$. The response in all cases is nonlinear with a slight increase up to about 15 cycles and then a rapid increase beyond 15 cycles. The increase in the absorbance suggests that initially nuclei formation dominates in the early cycles and then the nuclei grow into nanoparticles in the polymer multilayers with a larger number of 
the reductive hydrolysis reaction cycles of $\mathrm{Zn}^{2+}$ [11-15]. TEM images (see below) support this hypothesis. The results given in Figure 3(b) can be used to control the amount of nanoparticles deposited within the film by controlling the number of reaction cycles.

Transmission electron microscopy (TEM) is a very useful technique to investigate the presence, size and density of nanoparticles in the LbL film. A TEM image of a pristine PDDA/PSS polymer film (zero reaction cycles) of 6.5 LP is shown in Figure 4. The figure shows some contrast in the pristine LbL film mostly from spherical features, which gives a diffuse diffraction pattern due to their amorphous nature. The two types of polymers are held together in the LbL structure by coulombic forces which are dominant near the regions where the greatest concentration of opposite charges of the cationic and anionic polymer overlap. Presumably the contrast in the LbL polymer complex is caused denser amorphous regions due to interacting opposing clusters of charges.

TEM images of samples after 10 reaction cycles on 6.5 layers of polymers show a blunted arrow-like structures as seen for example in Figure 5. In contrast, samples below 10 cycles have very few of such structures (not shown). The pristine PDDA/PSS 6.5 LP film with zero reaction cycles has spherical features and these features interfere with the ability to observe small $\mathrm{ZnS}$ particles or nuclei for cycle numbers below 10 . The number density of the blunted arrow-like $\mathrm{ZnS}$ particles increases significantly after 10 cycles as shown in Figure 6 for 15 cycles. Comparing samples grown after 10 and 15 cycles, it appears that as the number of cycles increases, the number density of $\mathrm{ZnS}$ particles increases while their size (length) remains nearly constant $(200 \mathrm{~nm}-300 \mathrm{~nm})$. This suggests that each reaction cycle causes additional nuclei of $\mathrm{ZnS}$ to form, although the number of nuclei is relatively low per single cycle, while existing nuclei grow to uniform size. The TEM results agree well with UV-vis absorption data shown in Figure 3(b). Both figures indicate that the number of $\mathrm{ZnS}$ particles becomes significant at 10 cycles and beyond that number the growth and density of the $\mathrm{ZnS}$ nanoparticles increase with the number of cycles. Since the response in Figure 3(b) is nonlinear for larger numbers of cycles, it may be possible that the presence of nanoparticles facilitates the nucleation of more particles. Selected area electron diffraction (SAED) indicates a cubic spheralite structure of $\mathrm{ZnS}$ (inset Figure 6) [18].

\section{Conclusions}

The layer-by-layer technique has been used to deposit polyions as nanofilms on substrates. The films were pre-

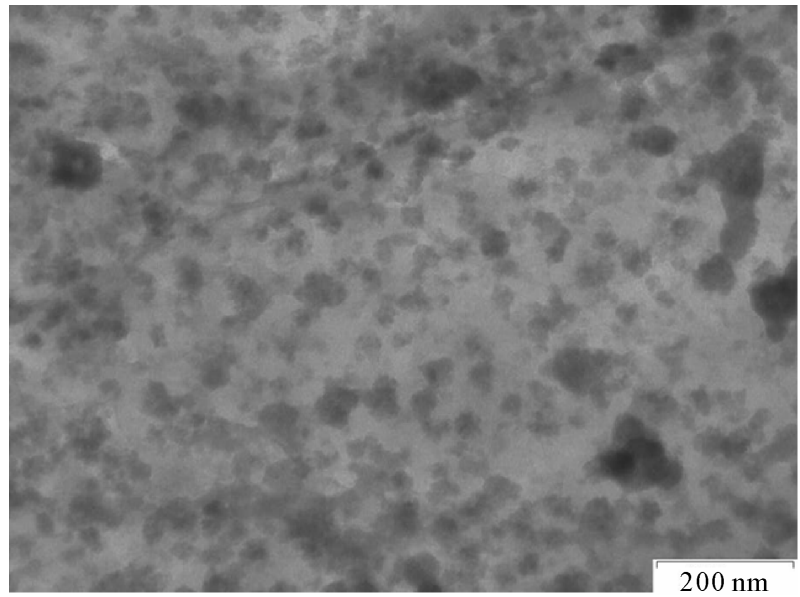

Figure 4. TEM micrograph of a PDDA/PSS sample (6.5 layer pairs). Bar is $200 \mathrm{~nm}$.

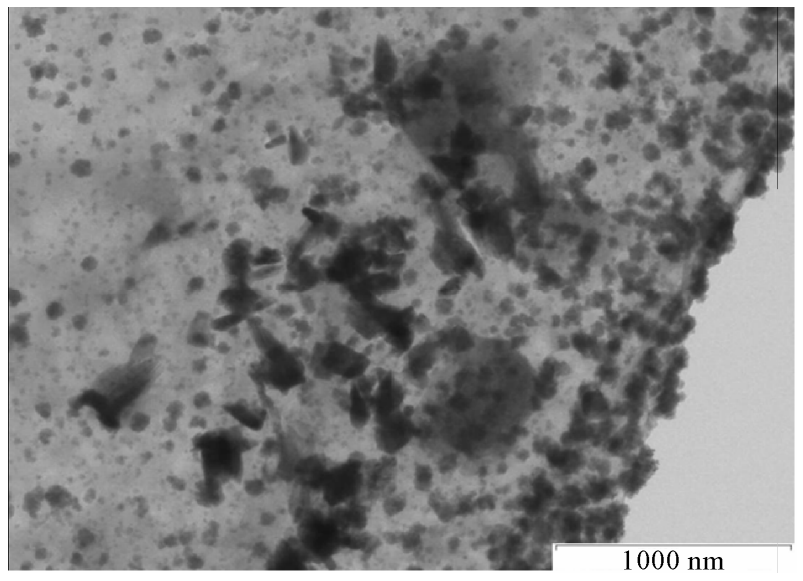

Figure 5. TEM image of 6.5 layer pairs after 10 cycles of reductive hydrolysis. Bar is $1000 \mathrm{~nm}$.

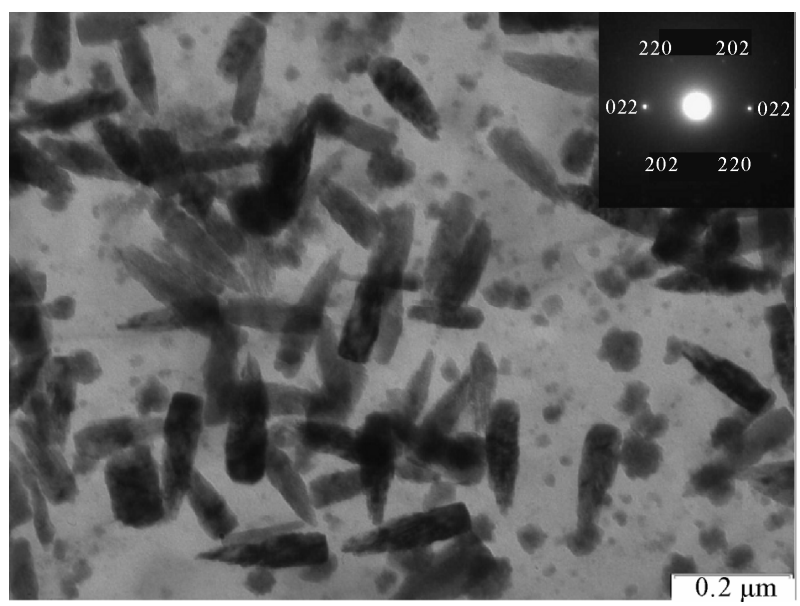

Figure 6. TEM image of 6.5 layer pairs after 15 cycles of reductive hydrolysis. The nucleated zinc sulfide particles have grown in size. Bar is 0.2 microns. The inset shows the selected area electron diffraction (SAED). 
pared by adsorbing a polycation and then a polyanion alternately on quartz substrates. Zinc sulfide nanoparticles were nucleated inside the films by way of a cyclic repetition of reductive hydrolysis. UV-visible spectroscopy shows a regular assembly of the polymer films and also indicates the subsequent nucleation and growth of particles in the polymer matrix by reductive hydrolysis. TEM studies confirm the presence and growth of $\mathrm{ZnS}$ nanoparticles, with cubic spheralite structure, in the LbL polymer matrix.

\section{Acknowledgements}

This work was supported by the Scientific and Technological Research Council of Turkey with International Post Doctoral Fellowship Program (2219) as a sabbatical fellowship to SC.

\section{REFERENCES}

[1] J. Lee, S. Lee, S. Cho, S. Kim, I. Y. Park and Y. D. Choi, "Role of Growth Parameters on Structural and Optical Properties of Zns Nanocluster Thin Films Grown by Solution Growth Technique," Materials Chemistry and Physics, Vol. 77, No. 1, 2002, pp. 254-260. doi:10.1016/S0254-0584(01)00563-6

[2] Y. Li, Y. Zhu, C. Li, X. Yang and C. Li, "Synthesis of Zns Nanoparticles into the Pore of Mesoporous Silica Spheres," Materials Letters. Vol. 63, No. 12, 2009, pp. 1068-1070. doi:10.1016/j.matlet.2009.02.007

[3] F. Göde, C. Gümüş and M. Zor, "Investigation on the Physical Properties of the Polycrystalline Zns Thin Films Deposited by the Chemical Bath Deposition Method," Journal of Crystal Growth, Vol. 299, No. 1, 2007, pp. 136-141. doi:10.1016/j.jcrysgro.2006.10.266

[4] T. L. Chu, S. S. Chu, J. Britt, C. Feredikes and C. Q. Wu, "Cadmium Zinc Sulfide Films and Heterojunctions," Journal of Applied Physics, Vol. 70, No. 5, 1991, P. 2688. doi:10.1063/1.349384

[5] T. B. Nasr, N. Kamoun and C. Guasch, "Physical Properties of ZnS Thin Films Prepared by Chemical Bath Deposition," Applied Surface Science, Vol. 254, No. 16, 2008, pp. 5039-5043. doi:10.1016/j.apsusc.2008.01.173

[6] P. Prathap, N. Revathi, Y. P. Venkata Subbaiah and K. T. Ramakrishna Reddy, "Thickness Effect on the Microstructure, Morphology and Optoelectronic Properties of Zns Films," Journal of Physics: Condensed Matter, Vol. 20, No. 3, 2008, p. 035205. doi:10.1088/0953-8984/20/03/035205

[7] Y. Wang, A. S. Angelatos and F. Caruso, "Template Synthesis of Nanostructured Materials Via Layer-By-Layer Assembly," Chemistry of Materials, Vol. 20, No. 3, 2008, pp. 848-858. doi:10.1021/cm7024813

[8] G. Yang, H. Ma, Z. Wu and P. Zhang, "Tribological behavior of ZnS-filled polyelectrolyte multilayers," Wear, Vol. 262, No. 3-4, 2007, pp. 471-476. doi:10.1016/j.wear.2006.06.018

[9] J. S. Ahn, P. T. Hammond, M. F. Rubner and I. Lee, "Self-Assembled Particle Monolayers on Polyelectrolyte Multilayers: Particle Size Effects on Formation, Structure, and Optical Properties," Colloids and Surfaces A: Physicochemical and Engineering Aspects, Vol. 259, 2005, pp. 45-53. doi:10.1016/j.colsurfa.2005.02.008

[10] S. Promnimit, C. Cavelius, S. Mathur and J. Dutta, "Growth of Gold/Zinc Sulphide Multilayer Films Using Layer-by- Layer Assembly of Colloidal Nanoparticles," Physica E, Vol. 41, 2008, pp. 285-291. doi:10.1016/j.physe.2008.07.013

[11] A. M. Fojas, E. Murphy and P. Stroeve, "Layer-by-Layer Polymeric Supramolecular Structures Containing Nickel Hydroxide Nanoparticles and Microcrystallites," Industrial \& Engineering Chemistry, Vol. 41, No. 11, 2002, pp. 2662-2667.

[12] L. Zhang, A. K. Dutta, G. Jarero and P. Stroeve, "Nucleation and growth of cobalt hydroxide crystallites in organized polymeric multilayers," Langmuir, Vol. 16, No. 17, 2000, pp. 7095-7100. doi:10.1021/1a000195o

[13] A. K Dutta, G. Jarero, L. Zhang and P. Stroeve, "In-Situ Nucleation and Growth of $\gamma$-FeOOH Nanocrystallites in Polymeric Supramolecular Assemblies," Chemistry of Material, Vol. 12, No. 1, 2000, pp. 176-181. doi:10.1021/cm990522h

[14] A. K. Dutta, T. Ho, L. Zhang and P. Stroeve, "Nucleation and Growth of Lead Sulfide Nano-and Microcrystallites in Supramolecular Polymer Assemblies," Chemistry of Material, Vol. 12, No. 4, 2000, pp. 1042-1048. doi:10.1021/cm990628c

[15] S. Dante, Z. Hou, S. Risbud and P. Stroeve, "Nucleation of Iron oxy-Hydroxide Nanoparticles by Layer-by Layer Polyionic Assemblies, Langmuir, Vol. 15, No. 6, 1999, pp. 2176-2182. doi:10.1021/la980587j

[16] C. Wang, E. Wang, Y. Lan, Q. Li, B. Mao and C. Tian, "Nucleation and Growth of Zno Nanocrystals in Polymer Films," Thin Solid Films, Vol. 516, 2008, pp. 6058-6062. doi:10.1016/j.tsf.2007.10.132

[17] G. Yang, Z-G Geng, H. Ma, Z. Wu and P. Zhang, "Preparation and Tribological Behavior of $\mathrm{Cu}$-Nanoparticle Polyelectrolyte Multilayers Obtained by Spin-Assisted Layer-By-Layer Assembly," Thin Solid Films, Vol. 517, 2009, pp. 1778-1783. doi:10.1016/j.tsf.2008.10.033

[18] M. Logar, B. Jančar, A. Rečnik and D. Suvorov, "Controlled Synthesis of Pure and Doped Zns Nanoparticles in Weak Polyion Assemblies: Growth Characteristics and Fluorescence Properties, Nanotechnology, Vol. 20, No. 27, 2009, article ID 275601. doi:10.1088/0957-4484/20/27/275601

[19] M. Logar, B. Jančar, A. Rečnik, D. Suvorov and R. Kostanjšek, "In situ synthesis of Ag nanoparticles in polyelectrolyte multilayers, Nanotechnology, Vol. 18, No. 32,2007 , article ID 325601 . doi:10.1088/0957-4484/18/32/325601

[20] T. B. Nasr, N. Kamoun and C. Guasch, "Structure, Sur- 
face composition, and Electronic Properties of Zinc Sulphide Thin Films," Materials Chemistry and Physics, Vol. 96 2006, pp. 84-89.

doi:10.1016/j.matchemphys.2005.06.051

[21] A. Antony, K. Y. Murali, R. Manoj and M. K. Jayaraj, "The Effect of the Ph Value on the Growth and Properties of Chemical-Bath-Deposition Zns Thin Films," Materials
Chemistry and Physics, Vol. 90 , 2005, pp. 06-110.

[22] Y. Lan, B. Mao, E. Wang, Y. Song, Z. Kang, C. Wang, C. Tian, C. Zhang, L. Xu and Z. Li, "In Situ Fabrication of Hybrid Polyoxometalate Nanoparticles Composite Films," Thin Solid Films, Vol. 515, 2007, pp. 3397-3401. doi:10.1016/j.tsf.2006.09.050 